Check for updates

Cite this: Chem. Commun., 2021, 57,5638

Received 29th October 2020, Accepted 16th March 2021

DOI: $10.1039 / \mathrm{d} 0 \mathrm{cc} 07165 \mathrm{j}$

rsc.li/chemcomm

\section{High shear spheroidal topological fluid flow induced coating of polystyrene beads with $\mathrm{C}_{60}$ spicules $\dagger$}

\author{
Matt Jellicoe, (iD Kasturi Vimalanathan, Jason R. Gascooke, (D) Xuan Luo (iD) and \\ Colin L. Raston (iD *
}

\begin{abstract}
Spheroidal spicular like topological fluid flow in an angled vortex fluidic device (VFD) housing a $20 \mathrm{~mm}$ diameter tube with a hemispherical base rotating at $4 \mathrm{k} \mathrm{rpm}$ and tilted at $45^{\circ}$ is effective in reducing the thermodynamic equilibrium concentration of fullerene $C_{60}$ in toluene, with the formation of spicules of the material under continuous flow processing. Under the same operational conditions in the presence of polystyrene beads 2 to $6 \mu \mathrm{m}$ in diameter, spicules of $C_{60} \mathrm{ca} .150 \mathrm{~nm}$ in length grow on their surfaces. This establishes that the spheroidal topological fluid flow in the VFD prevails while enveloping spheroidal like particles of such size.
\end{abstract}

Spheroidal like fullerene $\mathrm{C}_{60}$ comprised of $\mathrm{sp}^{2}$ carbon atoms arranged at the vertices of a truncated icosahedron $1 \mathrm{~nm}$ in diameter $^{1}$ can self-assemble into a variety of nano-structures, including rods, prisms, cones, spikes and vesicles. ${ }^{1-4}$ Such structures, depending on their size and morphology, have a variety of applications including in photodetectors, sensors, solar cells, light emitting diodes and drug delivery. ${ }^{2,5}$ In addition, chemically modified fullerene $\mathrm{C}_{60}$ has application for controlled slow release of encapsulated drugs. ${ }^{6}$ Recently, we established the use of the vortex fluidic device (VFD), Fig. 1, which is a thin film microfluidic platform, to control the selfassembly of the fullerene into rods, spicules, ${ }^{7}$ cones $^{8}$ and tubules, ${ }^{9}$ along with spheroidal entrapment of nano-meter size particles of self-assembled $\mathrm{C}_{60}$ by graphene, generated from graphite as an in situ process. ${ }^{10}$ Understanding the mechanism of formation of the self-assembled structures, depending on the choice of rotational speed, solvent or mixtures of solvents, uniquely formed at a tilt angle of $45^{\circ}$, has been made possible by linking the outcome of a number of other materials processing in the device, along with determining the spatial arrangement of holes formed in thin films of polysulfone (PSF) coating the inner

Flinders Institute for Nanoscale Science and Technology, College of Science and Engineering, Flinders University, Bedford Park, South Australia, 5042, Australia. E-mail: colin.raston@flinders.edu.au; Tel: +61882017958

$\dagger$ Electronic supplementary information (ESI) available. See DOI: 10.1039/ d0cc07165j surface of the tube, and the so called signature of a liquid. ${ }^{7}$ The latter is the sum of the effects of the change in temperature, film thickness and mixing time as a function of rotational speed, $\omega .^{7}$ These collective studies established three different types of topological fluid flow in the VFD for a $20 \mathrm{~mm}$ OD (17.5 mm ID) quartz tube with a hemispherical base (and similarly for a $10 \mathrm{~mm}$ OD tube), Fig. 1(a), namely (i) Coriolis circular flow (diameter $=d_{\mathrm{C}}$ ) from the hemispherical base of the tube, (ii) double helical flow (diameter $=d_{\mathrm{DH}}$ ) arising from eddies generated by the Faraday waves combined with the Coriolis from the curvature along the tube, and (iii) spicular or spheroidal flow arising from the collapse of circular and double helical flow of the same diameter $\left(d_{\mathrm{C}}=d_{\mathrm{DH}}\right)$, Fig. 1 . The signature of toluene established a region of circular flow at $c a$. $3 \mathrm{k} \mathrm{rpm}$, transitioning to spicular flow at $3.8 \mathrm{k}$ $\mathrm{rpm}$, then double helical flow $>6 \mathrm{k} \mathrm{rpm}$. When toluene solutions of $\mathrm{C}_{60}$ significantly below their saturation level are processed in the VFD, shear stress induced crystallisation prevails, affording spicules at $4 \mathrm{k} \mathrm{rpm}$, rods $>6 \mathrm{k} \mathrm{rpm}$, and a mixture of rods and spicules at $6 \mathrm{k}$ rpm. These structures define the topological fluid flow at these speeds, noting that in the absence of shear stress, the nanoparticles slowly redissolve in toluene, back to thermodynamic equilibrium. ${ }^{7}$

Under spheroidal spicular topological flow, hereafter referred to as spicular flow, graphene oxide collapses into spheroidal structures, reversibly forming scrolls under circular flow, and flat sheets for double helical flow. ${ }^{7}$ Spicular flow essentially provides a non-directional shear stress environment, and we deemed that this topological fluid flow is important in being able to control the shear stress environment for selectively coating particles and manipulating microorganisms. We hypothesised that spherical like particles in the dynamic thin film in the VFD could be shrouded by spicular topological fluid flow, depending on the diameter of the particles. To this end, we selected micron sized poly(styrene-co-divinylbenzene) beads as the particles of choice for determining the ability to coat them with fullerene $\mathrm{C}_{60}$ spicules under the aforementioned shear stress induced crystallisation processing. ${ }^{7}$ Poly(styrene-co-divinylbenzene) beads themselves find extensive use in a number of applications which are likely to be 


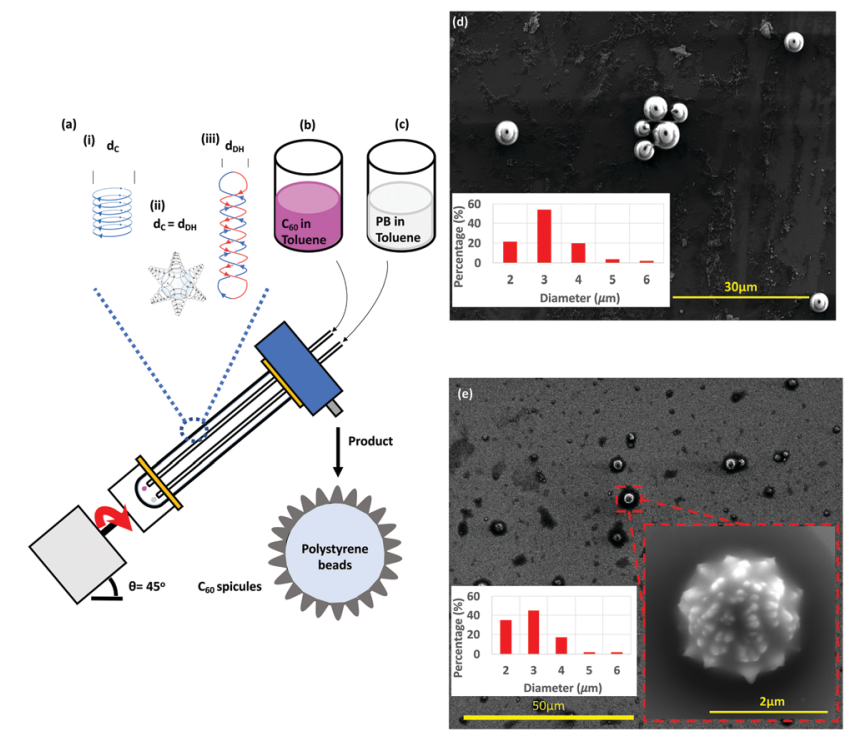

Fig. 1 Schematic illustration of the processing in the VFD and the associated topological fluid flows, (a), using a $1: 1$ mixture of a solution of $\mathrm{C}_{60}$ dissolved in toluene, (b), and a dispersion of poly(styrene-co-divinylbenzene) beads (PB) in toluene, (c). Scanning electron microscopy (SEM) images of the as-received beads processed in the absence of $\mathrm{C}_{60}$, with a statistical analysis of particle size $(2-6 \mu \mathrm{m}),(\mathrm{d})$, and coated beads formed at $\omega 4 \mathrm{k} \mathrm{rpm}$, flow rate $(\dot{\nu}) 0.1 \mathrm{~mL}$ $\min ^{-1}, \theta=45^{\circ}$, concentrations of $C_{60}$ and poly(styrene-co-divinylbenzene) in toluene $0.1 \mathrm{mg} \mathrm{mL}^{-1}$ with a zoomed in SEM image inset along with a statistical size distribution (2-6 $\mu \mathrm{m}),(\mathrm{e})$; SEM images were for samples prepared by drop-casting onto the surface of a silicon wafer. The different topological fluid flows, (a), for toluene in a $20 \mathrm{~mm}$ OD (17.5 mm ID) VFD tube, (i) Coriolis flow for $\omega>3 \mathrm{k} \mathrm{rpm}$, (ii) spicular flow for $\omega \mathrm{ca}$. $4 \mathrm{k} \mathrm{rpm}$ and (iii) double helical flow for $\omega>c a .6 \mathrm{k} \mathrm{rpm}{ }^{7}$

also relevant in the present and future studies, for example, in chromatography catalysis and cation exchange membranes. ${ }^{11,12}$ The present work is also of importance in further developing the utility of the VFD noting that it has a growing number and diversity of applications, including in controlling chemical reactivity and selectivity, protein folding, enhancing enzymatic reactions and separating proteins, ${ }^{13-15}$ and in materials processing. VFD-mediated material processing, beyond the above, include exfoliation of graphene from graphite and the manipulation of other 2D materials, ${ }^{10,16}$ creating toroidal structures of self-assembled single walled carbon nanotubes, ${ }^{17,18}$ and slicing single and multi-walled carbon tubes. ${ }^{19}$

In systematically exploring the operating parameters of the VFD while taking into account the fluid dynamic response of toluene in the VFD, as defined by its signature, ${ }^{7}$ we were able to coat spicules of $\mathrm{C}_{60} c a .150 \mathrm{~nm}$ in length, and $\geq c a .100 \mathrm{~nm}$ in diameter, over the surface of poly (styrene-co-divinylbenzene) beads $c a .2-6 \mu \mathrm{m}$ in diameter, Fig. 1 . Toluene solutions of $\mathrm{C}_{60}$ were initially prepared by adding as-received $\mathrm{C}_{60}$ to the solvent with the mixture allowed to stand overnight at room temperature, whereupon it was decanted to separate any residue. The concentration of $\mathrm{C}_{60}$ was then adjusted prior to the solution being delivered to the hemispherical base of the rapidly rotating quartz tube in the VFD through a jet feed, with a suspension of polystyrene beads in toluene $\left(0.1 \mathrm{mg} \mathrm{mL}^{-1}\right)$ delivered also at the base of the tube through a second jet feed, using separate syringe pumps, Fig. 1 . The choice of concentration of polystyrene beads was based on the concentration of $\mathrm{C}_{60}$ used for fabricating spicules of the fullerene in the absence of styrene beads, ${ }^{7}$ with higher concentrations potentially disturbing the topological fluid flow.

In a $20 \mathrm{~mm}$ OD tube in the VFD, toluene has primarily spicular or spheroidal like topological fluid flow at $\omega c a$. $4 \mathrm{k}$ rpm, whereas it is dominated by double helical flow for $\omega \geq 6 \mathrm{k}$ rpm. ${ }^{7}$ Prior to continuous flow processing in the VFD, we used the confined mode of operation where a finite amount of liquid $(\leq 2 \mathrm{~mL})$ was processed with the tube rotating at $4 \mathrm{k}$ or $7.5 \mathrm{k}$ rpm, with the concentration of $\mathrm{C}_{60}$ and polystyrene beads then varied. However, there was minimal coating of the polystyrene beads with $\mathrm{C}_{60}$ at both rotational speeds; at $4 \mathrm{k} \mathrm{rpm}$ for $0.1 \mathrm{mg} \mathrm{mL}{ }^{-1}$ concentrations of both materials, Fig. S5 (ESI $\dagger$ ), there are some $\mathrm{C}_{60}$ particles adhering to the isolated beads post VFD processing. Following this we moved to continuous flow operation of the VFD with the liquids introduced through jet feeds as discussed above. Here the flow rate $(\dot{\nu})$ was controlled using syringe pumps, noting that $0.1 \mathrm{~mL} \mathrm{~min}^{-1}$ is effective in generating spicules of fullerene $\mathrm{C}_{60}$ in the absence of polystyrene beads when the tube is rotating at $4 \mathrm{k} \mathrm{rpm}$, and tilted at $45^{\circ}$ $(\theta) .^{7}$ Now in the presence of such beads, using the same operating parameters and concentrations, coating of the beads with spicules of $\mathrm{C}_{60}$ was effective, for beads $c a$. 2-6 $\mu \mathrm{m}$ in diameter, Fig. 1. Of note is that any nanomaterials of $\mathrm{C}_{60}$ formed in the VFD in toluene arising from the induced shear stress crystallisation will redissolve slowly back to thermodynamic equilibrium, but with enough time for isolating them. ${ }^{7}$ Given that $\mathrm{C}_{60}$ solubilization in toluene is inherently slow and that the structures obtained in the VFD are uniform is size and shape with no eroded features, ${ }^{7}$ any flow induced breakup phenomena would be minimal. ${ }^{22}$ The coating of the polystyrene beads with $\mathrm{C}_{60}$ at the optimised conditions is in high yield ( $>98 \%$ ), with a 1:1 ratio of polystyrene and $\mathrm{C}_{60}$ having all the beads coated and without the formation of spicules devoid of the beads. Minor components of partially coated and/or fused beads also coated with $\mathrm{C}_{60}$ were observed, Fig. S4 (ESI $\dagger$ ).

The dimensions of the particles and properties were studied using scanning electron microscopy (SEM), energy-dispersive X-ray spectroscopy (EDX), X-ray diffraction (XRD), thermogravimetric analysis (TGA) and Raman spectroscopy (532 nm excitation wavelength). SEM was used to ascertain the size and shape of the material, as shown for the spherical spikes, Fig. 1. EDX revealed the material is essentially carbon with some surface oxidation, Fig. 2c. The Raman spectra showed the vibrational signature modes for the polystyrene beads (531, $980,1200,1603,2911$ and $3050 \mathrm{~cm}^{-1}$ ), and a broad fluorescence peak akin to the presence of $\mathrm{C}_{60}$, Fig. $2 \mathrm{f}^{20}{ }^{20}$ Interestingly, optical microscopy revealed that even when using the low laser power $(<0.01 \mathrm{~mW})$ in the Raman studies, the spicules are damaged during the spectroscopic studies.

The XRD data for the coated beads and as received $\mathrm{C}_{60}$ powder are in agreement with the database for the fcc phase of the fullerene, with the major peaks at $10.8^{\circ}, 17.7^{\circ}$, and $20.8^{\circ}$ corresponding to the (111), (220), and (311) planes respectively. ${ }^{21}$ However, the peaks are broad in the coated beads with the Scherrer equation giving an average crystal 

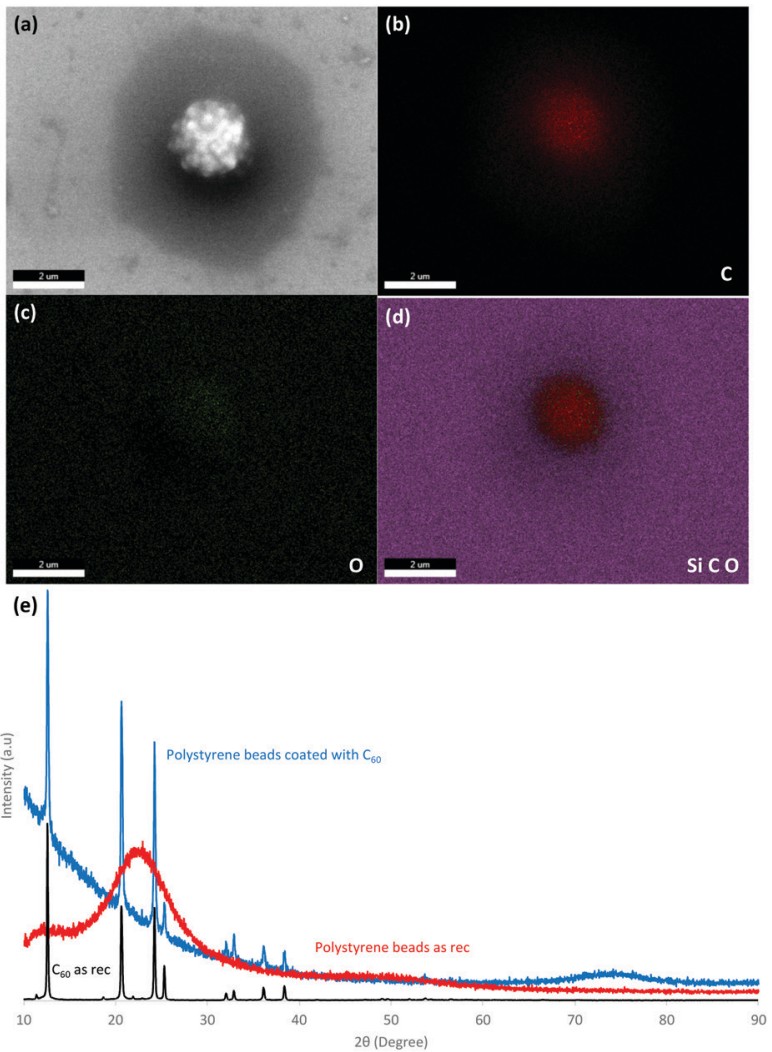

(f)

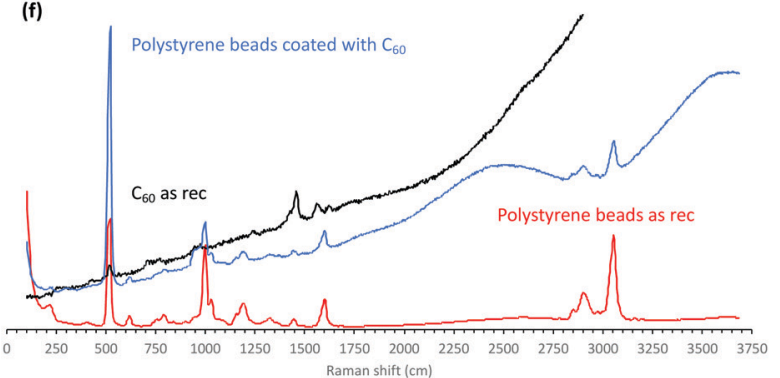

Fig. 2 Characterisation of the $C_{60}$ coated polystyrene beads formed

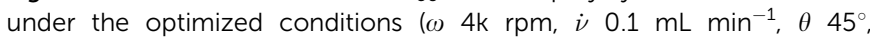
concentrations $0.1 \mathrm{mg} \mathrm{mL}^{-1}$ ) (a-d) SEM and EDX analysis (e) XRD patterns of the product (blue), as received poly(styrene-co-divinylbenzene) (red) and as received fullerene $C_{60}$ (black) and the Raman spectra (f) of the product (blue), fullerene $\mathrm{C}_{60}$ as received (red) and the as received poly(styrene-co-divinylbenzene) (black).

domain size of $28 \mathrm{~nm}$. The presence of small domains is consistent with other structures of self-assembled $\mathrm{C}_{60}$ generated in the VFD, including cones $c a .1 \mu \mathrm{m}$ in diameter, where the domains are $6.5 \mathrm{~nm}$ in diameter. ${ }^{8}$ In addition, spicules of $\mathrm{C}_{60}$ formed in the absence of polystyrene beads, or in the presence of beads which are too large for the topological fluid flow to coat them (see below), domains are also present, $6.5 \mathrm{~nm}^{8}$ and $27.1 \mathrm{~nm}$ in diameter, Fig. 2, respectively.

The presence of $\mathrm{nm}$ size domains in the shear stress induced crystallised $\mathrm{C}_{60}$ supports a two-step mechanism of growth, i.e. the generation of nanoparticles as the primary process, followed by their self-assembly into the different structures, depending on the topological fluid flow and the choice of solvent system, noting that cones are formed from shear stress generated in double helical flow, whereas for the spicules and spicule coated polystyrene beads, the fluid flow is a combination of this with the circular Coriolis flow from the hemispherical base of the rotating tube. ${ }^{7}$ The shear stress in the VFD is not uniform in the thin film of liquid but rather it can be uniformly located. ${ }^{7}$ The induced crystallization of $\mathrm{C}_{60}$, in coating polystyrene beads or in forming free standing material, is in accord with theoretical predicted shear driven aggregation of colloidal systems. ${ }^{23}$

Thermal gravitational analysis (TGA) under nitrogen was recorded for the $\mathrm{C}_{60}$ coated beads (stored for one week at room temperature) and compared with the as-received beads ( $c a .2-6 \mu \mathrm{m}$ ). The latter revealed a $95 \%$ weight loss between $300{ }^{\circ} \mathrm{C}$ and $440{ }^{\circ} \mathrm{C}$, Fig. S7a (ESI $\dagger$ ). The TGA profile for the composite material was dramatically different, with an increase in weight at $c a .120{ }^{\circ} \mathrm{C}$, which corresponds to oxidation of fullerene $\mathrm{C}_{60}$, followed by a sharp weight loss at $c a .380{ }^{\circ} \mathrm{C}$, although this is now less pronounced for increasing temperatures. Coating the $\mathrm{C}_{60}$ spicules on the surface of the beads increases the decomposing temperature profile. This is consistent with the coating of the polystyrene beads with $\mathrm{C}_{60}$ 'shielding' the polystyrene beads from the heat generated during the TGA. ${ }^{24,25}$

To ascertain the effect of the choice of operating parameters of the VFD for coating the polystyrene beads, and the importance of the associated topological fluid flows, we altered the tilt angle from $+45^{\circ}$ to +40 and $+50^{\circ}$ for beads 2 to $6 \mu \mathrm{m}$ in diameter. For the lower tilt angle, there is more agglomeration of the beads but nevertheless they are coated with spicules, Fig. 3(a and b). This also prevails for the higher tilt angle but with fibres connecting some of the beads indicating more complex fluid flow, Fig. 3(c and d). We then fixed the tilt angle at $45^{\circ}$ and used larger polystyrene beads, $c a$. $14-20 \mu \mathrm{m}$ in

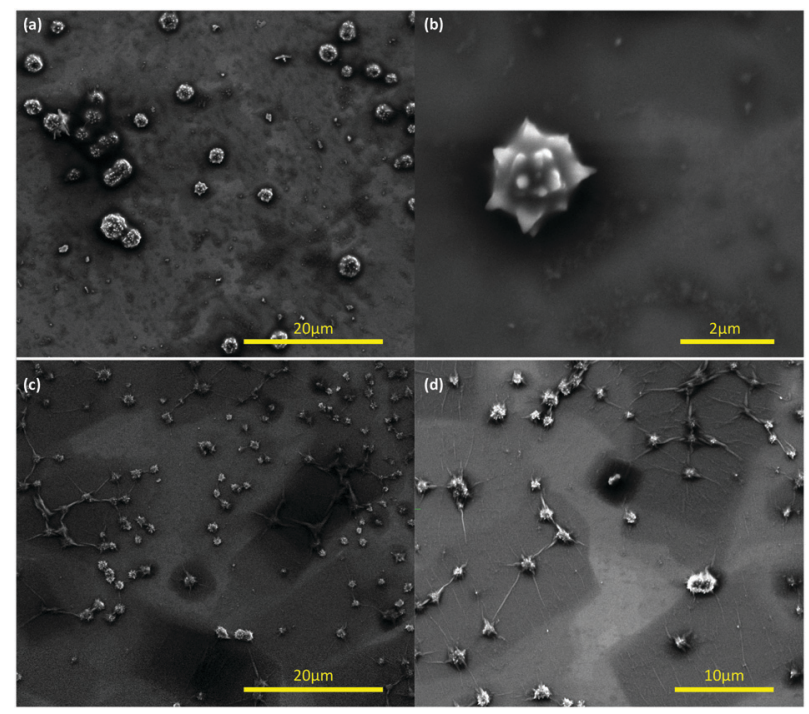

Fig. 3 SEM images of the composite material of $C_{60}$ and polystyrene beads drop casted onto the surface of a silicon wafer with the beads formed using the same processing conditions as in Fig. 1 but with the tilt angle $\theta$ set at ( $a$ and $b$ ) $40^{\circ}$, and ( $c$ and d) $50^{\circ}$. 

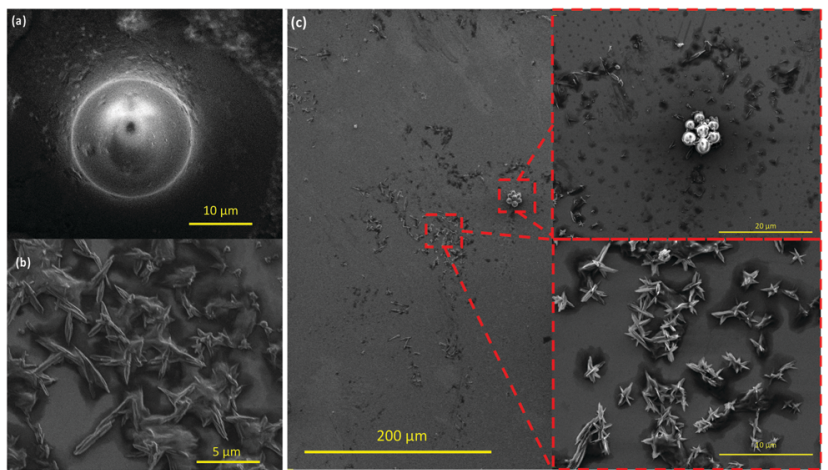

Fig. 4 SEM images ( $a$ and $b$ ) of the uncoated polystyrene beads (ca. 14-20 $\mu \mathrm{m}$ in diameter) and spicules of $C_{60}$ devoid of a polystyrene core, formed under the optimised conditions for coating 2-6 $\mu \mathrm{m}$ beads, Fig. 1. (c) Uncoated polystyrene beads (ca. 2-6 $\mu \mathrm{m}$ diameter) along with spicules of $\mathrm{C}_{60}$ devoid of a polystyrene core formed under the optimised conditions, Fig. 1, but now using a $10 \mathrm{~mm}$ OD VFD.

diameter, with the other VFD operating parameters the same as for optimal coating of the smaller breads, Fig. 1. Here there was no evidence for coating the beads, being unperturbed relative to the as received beads, with the $\mathrm{C}_{60}$ forming spicules comprised only of the fullerene, Fig. 4(a and b), as if the beads were not present. ${ }^{7}$ Thus there is a limit on the size of the beads that can be coated in a $20 \mathrm{~mm}$ OD diameter VFD tube, i.e. there is an upper limit on the ability for the spheroidal topological fluid flow to be able to accommodate a particle within its confines.

The diameter of the tube in the VFD can be varied and this processing option has been used in materials processing. ${ }^{7,16}$ In testing the effect of the choice of diameter on the nature of the product herein, we explored the possibility of coating polystyrene beads in a $10 \mathrm{~mm}$ OD tube. This is the original diameter of the tube used in developing the VFD. ${ }^{13}$ Reducing the diameter of the tube reduces the diameter of the topological fluid flow in the VFD. ${ }^{7}$ Translating the optimised conditions in Fig. 1 for a $20 \mathrm{~mm}$ OD tube for $2-6 \mu \mathrm{m}$ beads to a $10 \mathrm{~mm}$ OD tube for the same beads resulted in the formation of spicules of $\mathrm{C}_{60}$ devoid of a polystyrene core, the beads being devoid of surface bound spicules, Fig. 4(c). Thus, the spicular fluid flow in the $10 \mathrm{~mm}$ OD (7.5 mm ID) tube is too small to accommodate polystyrene beads $c a$. 2-6 $\mu \mathrm{m}$ in diameter.

In conclusion, we report a simple and effective method for fabricating fullerene $\mathrm{C}_{60}$ spicules over the surface of spherical poly(styrene-co-divinylbenzene) beads under continuous flow mode of operation of the VFD, and that there is an upper limit of the size of the beads that can be confined within the spheroidal topological fluid flow, $\geq c a$. $6 \mu \mathrm{m}$. The structures of the spheroidal composite material are uniform in shape, and importantly the isolated yield of the product is high $(>98 \%)$, with the processing scalable under continuous flow conditions. The ability to selectively coat a certain diameter particle lends itself for size specific surface modification for applications in materials processing, and also in the coating of small molecule drugs, nanoparticles, and macromolecules over the surface of micron sized particles that can be accommodated in the spheroidal topological fluid flow. Further development of this processing to control the thickness of any coating is also important for specific applications.

Matt Jellicoe thanks Flinders University for scholarship funding, and the authors gratefully acknowledge financial support from the Australian Research Council. The authors acknowledge the expertise, equipment and support provided by Microscopy Australia (MA) and the Australian National Fabrication Facility (ANFF) at the South Australian nodes under the National Collaborative Research Infrastructure Strategy.

\section{Conflicts of interest}

There are no conflicts to declare.

\section{Notes and references}

1 M. Prato, J. Mater. Chem., 1997, 7, 1097-1109.

2 M. Sathish, K. i. Miyazawa, J. P. Hill and K. Ariga, J. Am. Chem. Soc., 2009, 131, 6372-6373.

3 M.-S. Lin, R.-T. Chen, N.-Y. Yu, L.-C. Sun, Y. Liu, C.-H. Cui, S.-Y. Xie, R.-B. Huang and L.-S. Zheng, Colloids Surf., B, 2017, 159, 613-619.

4 T. Konno, T. Wakahara and K. I. Miyazawa, J. Cryst. Growth, 2015, 416, 41-46.

5 K. i. Miyazawa, J.-i. Minato, T. Yoshii and T. Suga, Sci. Technol. Adv. Mater., 2005, 6, 388-393.

6 K. Ariga, Q. Ji, J. P. Hill, Y. Bando and M. Aono, NPG Asia Mater., $2012,4, \mathrm{e} 17$.

7 T. M. D. Alharbi, et al., n.d., DOI: 10.26434/chemrxiv.13141352.v1.

8 I. K. Alsulami, T. M. D. Alharbi, D. P. Harvey, C. T. Gibson and C. L. Raston, Chem. Commun., 2018, 54, 7896-7899.

9 K. Vimalanathan, R. G. Shrestha, Z. Zhang, J. Zou, T. Nakayama and C. L. Raston, Angew. Chem., Int. Ed., 2017, 56, 8398-8401.

10 X. Chen, J. F. Dobson and C. L. Raston, Chem. Commun., 2012, 48, 3703-3705.

11 Q. C. Wang, F. Švec and J. M. J. Fréchet, J. Chromatogr. A, 1994, 669, 230-235.

12 S. Savari, S. Sachdeva and A. Kumar, J. Membr. Sci., 2008, 310, 246-261.

13 L. Yasmin, X. Chen, K. A. Stubbs and C. L. Raston, Sci. Rep., 2013, 3, 2282.

14 J. Britton, R. P. Dyer, S. Majumdar, C. L. Raston and G. A. Weiss, Angew. Chem., Int. Ed., 2017, 56, 2296-2301.

15 J. Britton, L. M. Meneghini, C. L. Raston and G. A. Weiss, Angew. Chem., Int. Ed., 2016, 55, 11387-11391.

16 A. H. M. Al-antaki, T. M. D. Alharbi, S. Kellici, N. P. Power, W. Lawrance and C. L. Raston, ChemNanoMat, 2020, 6, 657-662.

17 K. Vimalanathan, X. Chen and C. L. Raston, Chem. Commun., 2014, 50, 11295-11298.

18 T. M. D. Alharbi, Y. Shingaya, K. Vimalanathan, T. Nakayama and C. L. Raston, ACS Appl. Nano Mater., 2019, 2, 5282-5289.

19 K. Vimalanathan, J. R. Gascooke, I. Suarez-Martinez, N. A. Marks, H. Kumari, C. J. Garvey, J. L. Atwood, W. D. Lawrance and C. L. Raston, Sci. Rep., 2016, 6, 22865.

20 T. Shimada, Y. Yamamoto, T. Kaji, K. Itaka, H. Koinuma, Y. Kuninobu, Y. Matsuo, E. Nakamura and K. Saiki, Solid State Commun., 2004, 132, 197-201.

21 K. Miyazawa, Y. Kuwasaki, A. Obayashi and M. Kuwabara, J. Mater. Res., 2002, 17, 83-88.

22 F. Mura and A. Zaccone, Phys. Rev. E, 2016, 93, 042803.

23 A. Zaccone, H. Wu, D. Gentilli and M. Morbibelli, Phys. Rev. E: Stat., Nonlinear, Soft Matter Phys., 2009, 80, 051404.

24 I. K. Alsulam, T. M. D. Alharbi, M. Moussa and C. L. Raston, ACS Omega, 2019, 4, 19279-19286.

25 F.-A. Teng, Y. Guo, H. Jianping, Y. Zhang and Z. Han, Des. Monomers Polym., 2017, 20, 283-292. 